\title{
Nonunitary triplet pairing in the noncentrosymmetric superconductor $\mathrm{LaNiC}_{2}{ }^{\star}$
}

\author{
Gábor Csire ${ }^{1, a}$, Balázs Újfalussy ${ }^{2}$, and James F. Annett ${ }^{1}$ \\ ${ }^{1}$ H. H. Wills Physics Laboratory, University of Bristol, Tyndall Ave, Bristol BS8-1TL, UK \\ 2 Institute for Solid State Physics and Optics, Wigner Research Centre for Physics, Hungarian Academy of Sciences, \\ PO Box 49, 1525 Budapest, Hungary
}

Received 26 February 2018 / Received in final form 18 June 2018

Published online 1 October 2018

(C) The Author(s) 2018. This article is published with open access at Springerlink.com

\begin{abstract}
We present a first-principles based semiphenomenological approach to study the superconductivity in the noncentrosymmetric superconductor $\mathrm{LaNiC}_{2}$ with spin-orbit coupling. Based on group theoretical considerations, it was already shown that the breaking of time-reversal symmetry is only compatible with nonunitary triplet pairing states. To investigate the pairing mechanism by which this comes about and the possible role of the lack of inversion symmetry, we have combined the relativistic spin-polarized version of Korringa-Kohn-Rostoker method for the solution of the Dirac-Bogoliubov-de Gennes equations with a semiphenomenological parametrization of the pairing interaction. This made possible to study different orbital specific pairing models in quantitative details. We compare our predictions for the temperature dependence of the specific heat and it is found that it can be described by an interorbital equal-spin pairing on the nickel which breaks the time-reversal symmetry.
\end{abstract}

Noncentrosymmetric superconductors have been a subject of great interest since the discovery of superconductivity in the heavy-fermion material $\mathrm{CePt}_{3} \mathrm{Si}$ [1]. The main consequence of broken inversion symmetry in superconductors is that it admits the possibility of mixed spin singlet and spin triplet pairing. In this paper, we focus on $\mathrm{LaNiC}_{2}$ which is an unconventional noncentrosymmetric superconductor with $T_{c}=2.7 \mathrm{~K}$. The unconventional behavior was observed through zero-field muon spin resonance which indicated time-reversal symmetry breaking at the superconducting instability [2]. There are a number of other examples of time-reversal symmetry breaking superconductors including for example (U, Th) $\mathrm{Be}_{13}$ [3], $\mathrm{Sr}_{2} \mathrm{RuO}_{4}$ [4], $\mathrm{UPt}_{3}$ [5], (Pr, La)(Ru, Os) ${ }_{4} \mathrm{Sb}_{12}$ [6,7], $\mathrm{PrPt}_{4} \mathrm{Ge}_{12}$ [8], LaNiGa 2 [9], SrPtAs [10], $\mathrm{Re}_{6}(\mathrm{Zr}, \mathrm{Hf}, \mathrm{Ti})$ [11-13], $\mathrm{Lu}_{5} \mathrm{Rh}_{6} \mathrm{Sn}_{18}$ [14], $\mathrm{La}_{7} \mathrm{Ir}_{3}$ [15], and interestingly, twin-boundary interfaces of FeSe [16].

Although evidence for nodal gap structure was found from some measurements [17-19], recent specific heat [20,21], nuclear quadrupole relaxation [22], magnetic impurity [23], and penetration depth measurements [24] indicate fully gapped behavior in $\mathrm{LaNiC}_{2}$. It was also measured that the magnetization points in the direction of the $z$-axis indicating close relationship with the crystal structure, which lacks inversion symmetry along the $z$-axis [25].

\footnotetext{
* Contribution to the Topical Issue "Special issue in honor of Hardy Gross", edited by C.A. Ullrich, F.M.S. Nogueira, A. Rubio, and M.A.L. Marques.

a e-mail: csire.gab@gmail.com
}

For $\mathrm{LaNiC}_{2}$ symmetry analysis implies that the superconducting instability is of the non-unitary triplet type, with a spin-orbit coupling that is comparatively weak leading to a small singlet component [26]. However, the microscopic nature of the pairing mechanism leading to broken time-reversal symmetry is still a mystery. In order to understand the nature of superconductivity, we apply the semiphenomenological strategy suggested by Gyorffy et al. in reference [27] which is based on a combination of density-functional theory (DFT) within the local density approximation (LDA) and the Bogoliubov-de Gennes (BdG) equations. The goal is to determine the local orbitals which the electrons occupy when they are involved in the pairing interaction. Here, we employ a fully relativistic description based on the Dirac-Bogoliubov-de Gennes (DBdG) equations $[28,29]$ combined with a particularly efficient representation of the electron-electron interaction afforded by the DFT of superconductivity. In practice, this progress made possible by the recent generalization of the screened Korringa-Kohn-Rostoker (KKR) method for the solution of the DBdG equations presented in reference [30]. In this way, we incorporate every details of the band structure (including the effect of spin-orbit coupling) in the calculations, while the pairing interaction is described by phenomenological interaction parameters. We point out that the symmetry of the resulting gap is not assumed, but is the direct consequence of the assumed pairing model. For sake of completeness we should also mention that a non-relativistic spin DFT 


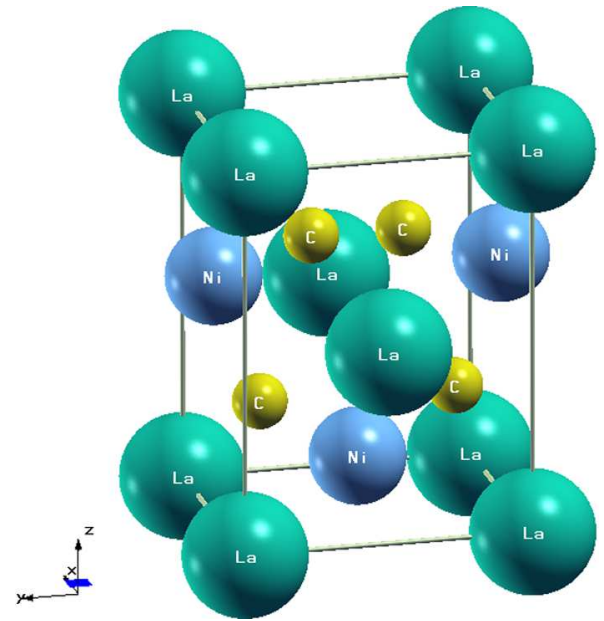

Fig. 1. Crystal structure of $\mathrm{LaNiC}_{2}$ (conventional unit cell).

for superconductors and the approximated exchangecorrelation functionals have been developed by Linscheid et al. [31,32]. At present, this is the most accurate theory, which allows the first principles calculation of the superconducting transition temperature for bulk systems [33-40]. However, despite the simplicity of the LDA + BdG approximation [27], it was able to describe the quasiparticle spectrum of cuprates $[41,42]$, and the proximity effect in superconducting heterostructures [43-45].

The crystal structure of $\mathrm{LaNiC}_{2}$ is simple (base centered orthorhombic structure with Amm2 space group) but noncentrosymmetric as shown in Figure 1. The La atoms form trigonal prisms, which are alternately filled by $\mathrm{Ni}$ and $\mathrm{C}$ dimers, hence breaking the inversion symmetry along $z$-axis as it can be seen in Figure 1 . The normal state electronic structure was already published several times (see Refs. [46-50]). Hence, here we just briefly review the important properties. The electronic structure calculations were performed with the KKR-method and the Quantum Espresso code [51]. We used the experimentally reported lattice parameters $a=3.952 \AA, b=4.557 \AA$, $c=6.193 \AA$, with the following fractional coordinates La $(0,0,0), \mathrm{Ni}(0.5,0,0.626)$, and $\mathrm{C}(0.5,0.160,0.289)[52]$. Without spin-orbit coupling there are two bands that cross the Fermi level and they have mixed $\mathrm{Ni} 3 d$, La $5 d$, and $\mathrm{C} 2 p$ characters. One of them is nearly halffilled showing a large dispersion along the $z$-axis, while the other band gives a small electron pocket. Spin-orbit coupling leads to the splitting of these sheets consistent with the noncentrosymmetric structure. The calculated Fermi surface of $\mathrm{LaNiC}_{2}$ is shown in Figure 2. As it can be seen the effect of spin-orbit coupling is not so large. The reported average value of the splitting is $3.1 \mathrm{mRy}$ [48], which is about half of that in $\mathrm{CePtSi}_{3}$ [1]. The Fermi surface has much weaker nesting properties compared to $(\mathrm{Sm}, \mathrm{Gd}, \mathrm{Nd}) \mathrm{NiC}_{2}$ which explains the absence of a charge-density wave [47].

We have found that the majority of the $\mathrm{Ni} d$ character in the density of states (DOS) is away from the Fermi level, however, there is still a significant $\mathrm{Ni} d$ contribution near $E_{F}$. The DOS has a relatively high value around
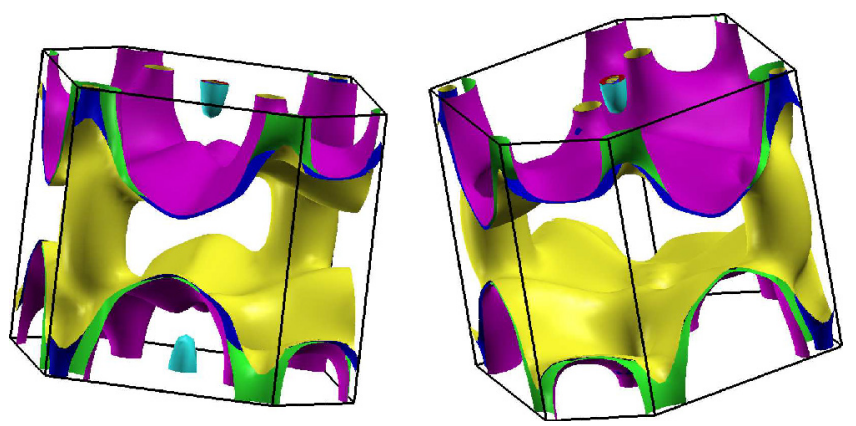

Fig. 2. Fermi surface of $\mathrm{LaNiC}_{2}$ (with spin-orbit coupling).

2.5 states $/ \mathrm{eV}$ around $E_{F}$, and the $\mathrm{Ni} d$ states cause a very small magnetic moment $0.001 \mu \mathrm{B}$ on the $\mathrm{Ni}$ atom. This magnetic moment disappears by assuming an artificial centrosymmetric structure in the calculations, hence can be related to the lack of inversion symmetry. Since many $\mathrm{RNiC}_{2}(\mathrm{R}=$ rare earth) compounds show antiferrogmagnetic behavior [53], the spin-polarized self-consistent calculations were also started from antiferrogmagnetic order by blowing up the unit cell, but it has ended in a ferromagnetic state. However, this does not provide clear evidence for a ferromagnetic order, since the proper way to determine the magnetic order of the ground state is to perform ab-initio spin dynamics at zero temperature [54]. Recently, an experimental hint of a magnetic phase was also found in reference [19] even for small pressure which seems to agree with our results.

Since the Fermi surface has a strong orbital degeneracy, and it is a very complex mixture of all $\mathrm{Ni} 3 d$, La $5 d$, and $\mathrm{C} 2 p$ orbitals, which makes a detailed tight-binding model description very difficult. Therefore, it is advisable to include all the details of the band structure in the solution of the DBdG equations which is possible with the DBdG-KKR method [30].

First, we investigate the possible role and importance of the spin-orbit coupling. The group theoretical results suggested that the singlet-triplet mixing due to the noncentrosymmetric crystal structure must be small. However, the $3.1 \mathrm{mRy}$ average value of the band splitting around the Fermi level looks quite significant compared to the typical values of the superconducting gap. Therefore, by assuming $\Delta=1 \mathrm{meV}$ conventional $s$-wave pairing, we calculated the triplet components with the DBdG-KKR method [30] as a function of the spin-orbit coupling scaling parameter (see Ref. [55]). To measure the effect of the spin-orbit coupling we associate charge-like quantities to the anomalous densities by integrating over the muffin-tin spheres. The same on-site $\Delta$ for every atoms creates fully gapped quasiparticle spectrum, with the ratio for the singlet components of $(\mathrm{La})$ 1:(Ni) 0.9:(C) 0.1, respectively, which is the consequence of the higher DOS at the Fermi level for the La, Ni atoms compared to the $\mathrm{C}$ atoms. As it can be seen from Figure 3 the spin-orbit coupling induces every type of triplet components in the site-resolved representation. However, the charges corresponding to the site-resolved equal-spin ( $\uparrow$ and $\downarrow \downarrow)$ triplet components are the same, which means that the time-reversal 

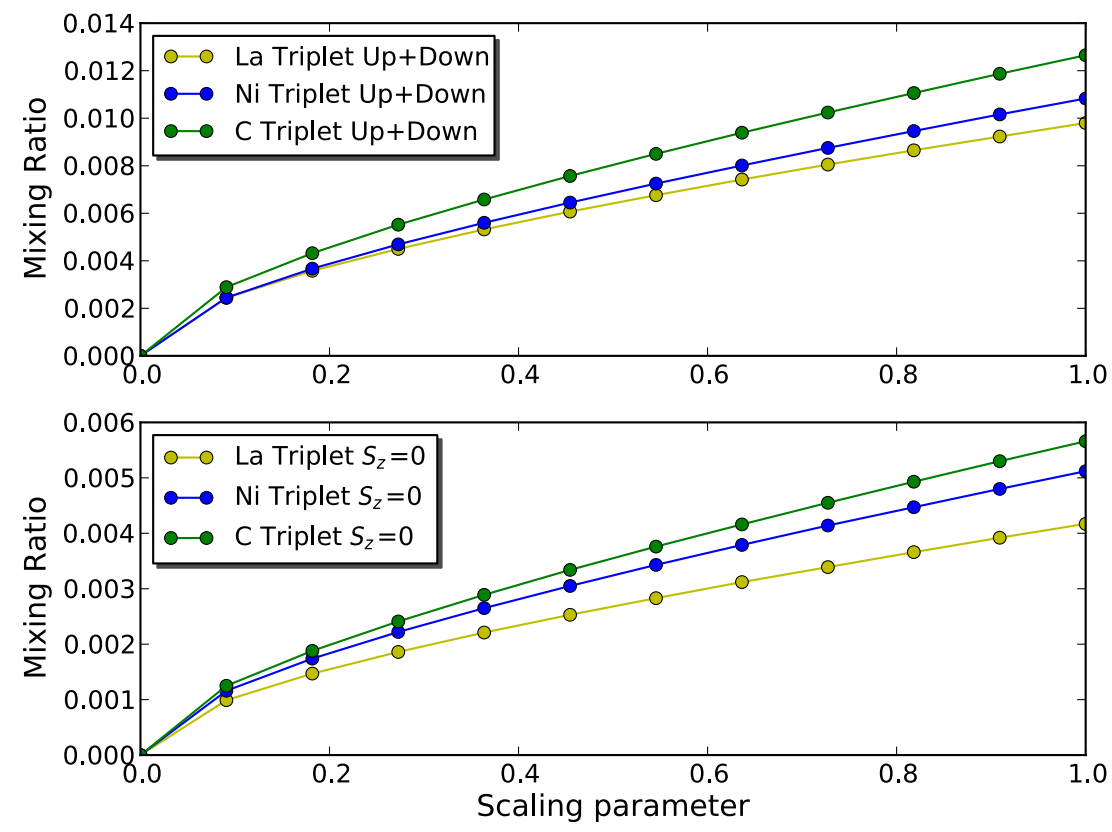

Fig. 3. Site-resolved singlet-triplet mixing ratios: the absolute value of the charge associated to the triplet component divided by the charge corresponding to the singlet component for every atoms.

symmetry is not broken. Moreover, the sum for equal-spin triplet components of all sites is zero, since a negative sign appears for the equal-spin triplet components on the $\mathrm{Ni}$ and $\mathrm{C}$ atoms, which is a restriction by symmetry [26]. Figure 3 also suggests that the effect of spin-orbit coupling on the pairing interactions is small, since the full singlet-triplet mixing ratio is $<2 \%$. This observation is in good agreement with the predictions made by symmetry investigations in reference [26].

To explain both time-reversal symmetry breaking, and fully gapped quasiparticle spectrum, here we employ the model originally developed for $\mathrm{LaNiGa}_{2}$ [56]. Since the singlet-triplet mixing effect is only modest, we shall use the concept of angular momentum and spin when we investigate the possible pairing interactions which could lead to time-reversal symmetry breaking state. While in the practical KKR calculations we change into the relativistic $|\kappa, \mu\rangle$ basis by a Clebsch-Gordan transformation. In reference [56], a novel triplet superconducting state was proposed, where the pairing occurs between electrons of the same spin on different orbitals. In certain cases (depending on the orbital character), this model can lead to a nodeless two-gap superconducting state which breaks time reversal symmetry. Now, it is instructive to assume an interorbital equal-spin pairing on the $\mathrm{Ni}$ atoms (see Fig. 4), since Ni $d$ states may be responsible for spontaneous magnetism observed in reference [25]. To describe such scenario we use a basis set of local orbitals $\left\{\phi_{L}\right\}$ to describe the single-particle wave function

$$
\left(\begin{array}{l}
u_{j}(\mathbf{r}) \\
v_{j}(\mathbf{r})
\end{array}\right)=\sum_{R, L} \phi_{L}(\mathbf{r}-\mathbf{R})\left(\begin{array}{l}
u_{P, j} \\
v_{P, j}
\end{array}\right),
$$

where $P=(R, L), R$ labels the site, and $L$ is the composite index including the angular quantum number $(\ell)$, the magnetic quantum number $(m)$, and the spin

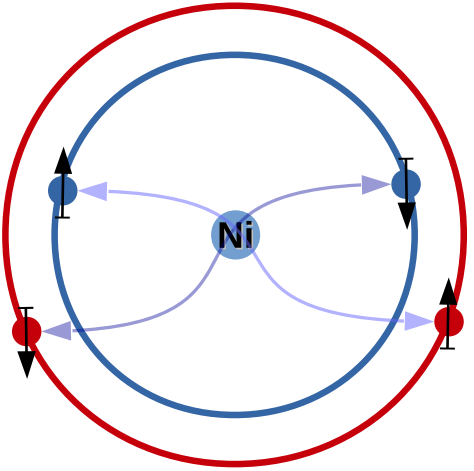

Fig. 4. Pairing model: interorbital equal-spin pairing on the $\mathrm{Ni}$ atoms.

index $(s)$. This leads to the DBdG equation in the orbital representation

$$
\sum_{P}\left(\begin{array}{cc}
H_{P^{\prime} P, D}-\varepsilon O_{P^{\prime} P} & D_{P^{\prime} P} \\
D_{P P^{\prime}}^{*} & -H_{P^{\prime} P, D}^{*}-\varepsilon O_{P^{\prime} P}
\end{array}\right)\left(\begin{array}{l}
u_{P, j} \\
v_{P, j}
\end{array}\right)=0
$$

where the Dirac Hamiltonian is

$$
\begin{aligned}
H_{P^{\prime} P, D}= & \int d \mathbf{r} \phi_{L^{\prime}}^{*}\left(\mathbf{r}-\mathbf{R}^{\prime}\right)\left[c \boldsymbol{\alpha} \mathbf{p}+\left(\boldsymbol{\beta}-\mathbb{I}_{4}\right) c^{2} / 2\right. \\
& \left.+\left(V_{\mathrm{eff}}(\mathbf{r})-E_{F}\right) \mathbb{I}_{4}+\boldsymbol{\Sigma} \vec{B}_{\mathrm{eff}}(\mathbf{r})\right] \\
& \times \phi_{L}(\mathbf{r}-\mathbf{R}),
\end{aligned}
$$

$V_{\text {eff }}$ and $B_{\text {eff }}$ are the effective electrostatic potential and exchange-field obtained from the normal state DFT calculations. The overlap matrix is defined as

$$
O_{P^{\prime} P}=\int d \mathbf{r} \phi_{L^{\prime}}^{*}\left(\mathbf{r}-\mathbf{R}^{\prime}\right) \phi_{L}(\mathbf{r}-\mathbf{R})
$$




$$
\boldsymbol{\alpha}=\left(\begin{array}{cc}
0 & \boldsymbol{\sigma} \\
\boldsymbol{\sigma} & 0
\end{array}\right), \quad \boldsymbol{\beta}=\left(\begin{array}{cc}
\mathbb{I}_{2} & 0 \\
0 & -\mathbb{I}_{2}
\end{array}\right), \quad \boldsymbol{\Sigma}=\left(\begin{array}{cc}
\boldsymbol{\sigma} & 0 \\
0 & \boldsymbol{\sigma}
\end{array}\right)
$$

and $\boldsymbol{\sigma}$ denotes the Pauli matrices. By assuming that the pairing interaction is local, it leads to the following self-consistency condition for the pairing potential

$$
\begin{aligned}
D_{P^{\prime} P}= & \Lambda_{P^{\prime} P} \int d \mathbf{r} \phi_{L^{\prime}}^{*}\left(\mathbf{r}-\mathbf{R}^{\prime}\right) \sum_{j}\left(1-2 f\left(\varepsilon_{j}\right)\right) \\
& \times \frac{u_{P, j}^{T} v_{P^{\prime}, j}^{*}+u_{P^{\prime}, j}^{T} v_{P, j}^{*}}{2} \phi_{L}(\mathbf{r}-\mathbf{R}) \\
= & \Lambda_{P^{\prime} P} \chi_{P^{\prime} P} .
\end{aligned}
$$

Here, the pairing potential is proportional to the corresponding component of the pairing amplitude $\chi_{P^{\prime} P}$ and is independent of the other components, and the interaction strengths $\Lambda_{P^{\prime} P}$ are treated as adjustable parameters. Our strategy is based on that if we choose only one of the interorbital interaction $\left(R=R^{\prime}\right.$, but $\left.L \neq L^{\prime}\right)$ to be nonzero, and fix the interaction strength by requiring that the transition temperature $T_{c}$ agrees with experimental one then all other superconducting properties can be calculated without further adjustable parameters. Particularly, we will calculate the specific heat. If one of these models do fit the experimental facts then we may conclude that, whatever physical mechanism is causing the pairing interaction, it acts between electrons occupying the orbitals selected in our model.

At the Fermi level the DOS contributions coming from all $5 d \mathrm{Ni}$ orbitals are significant, which means there are 10 possibilities for the interorbital equal-spin pairing model on the $\mathrm{Ni}$ atom. Again we assume the superconducting gap to be $1 \mathrm{meV}$ and our goal is to find out which gives fully gapped quasiparticle spectrum and nodal gap structure. To calculate the DOS in all of these superconducting states considerable extra effort was necessary (about $250000 k$-points in the two-dimensional Brillouin-zone) to obtain the crucial features (the behavior of the quasiparticle DOS around the Fermi level) which are related to the gap structure. In Figure 5, we can see that in most of the cases the DOS has V-shape around the Fermi level, which obviously implies line nodes in the quasiparticle spectrum. These slopes also determine the low-temperature specific heat as a function of temperature. However, equalspin pairing on the $d_{z^{2}}-d_{x^{2}-y^{2}}$ and $d_{z^{2}}-d_{x y}$ orbitals yields anisotropic fully gapped quasiparticle spectrum. Then, one obvious question is the relationship between this gap structure and the noncentrosymmetric crystal structure. Therefore, we have changed the fractional coordinates of the $\mathrm{Ni}$ atoms from the original $(0.5,0,0.626)$ to $(0.5,0,0.5)$, which transforms the $\mathrm{Ni}$ sublattice into centrosymmetric structure. Interestingly, the transformation of the lattice structure transformed the fully gapped behavior into nodal gap structure as it can be observed in Figure 6 . The same effect can also be achieved by scaling down spin-orbit coupling to zero, which indicates a close relationship between the splitting of the Fermi surface and the structure of the superconducting gap.
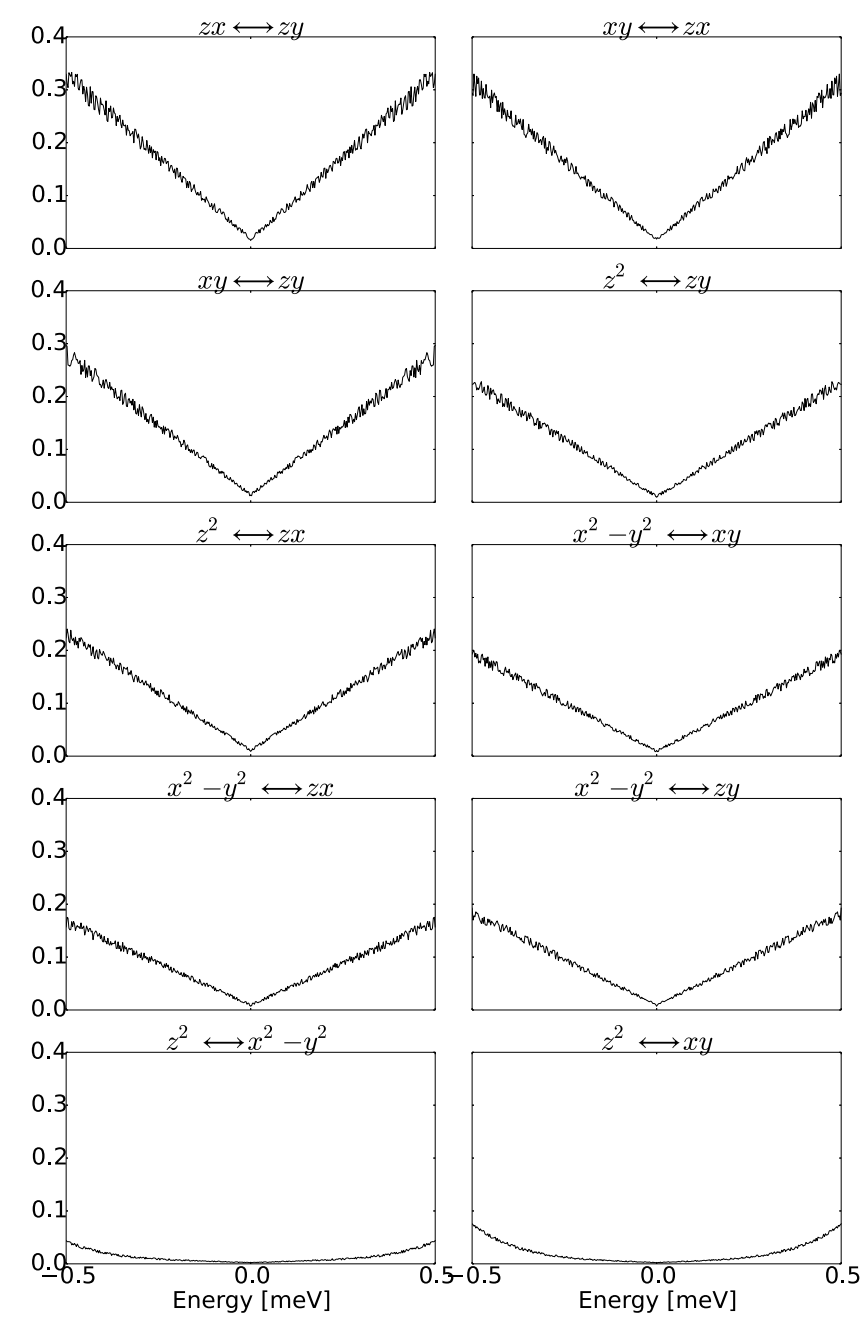

Fig. 5. Density of states (arbitrary units) in the superconducting state for different interorbital equal-spin pairing models on the $\mathrm{Ni}$ atom.

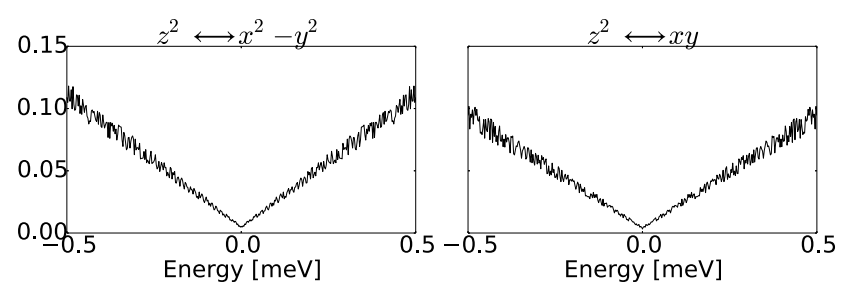

Fig. 6. Density of states (arbitrary units) in the superconducting state for different interorbital equal-spin pairing models on the $\mathrm{Ni}$ atom for the artificial crystal structure.

In the next step, we choose the two fully gapped scenarios (equal-spin pairing on the $d_{z^{2}}-d_{x^{2}-y^{2}}$ and $d_{z^{2}}-d_{x y}$ orbitals) and another one $\left(d_{z x}-d_{z y}\right)$ with nodal gap structure. We have obtained the strengths of the interactions for these cases by fitting to the experimental $T_{c}$ via a self-consistent solution of the DBdG

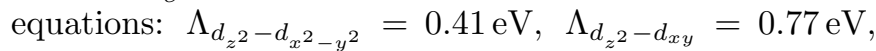
$\Lambda_{d_{z x}-d_{z y}}=2.4 \mathrm{eV}$. We can also conclude the cases with smaller interaction strength indicates that the particular pairing interaction is more likely to happen in nature. 


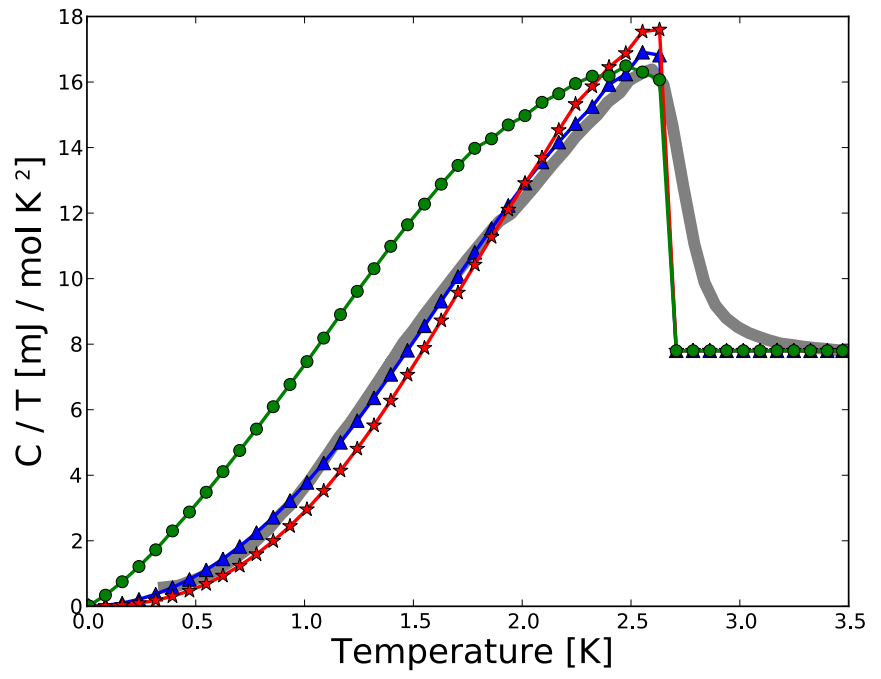

Fig. 7. Specific heat: the grey line is the experimental data taken from reference [21], the green line corresponds to equalspin pairing model on the $d_{z x}-d_{z y}$ orbitals, the red line refers to the pairing on the $d_{z^{2}}-d_{x y}$ orbitals, and the blue line indicates pairing on the $d_{z^{2}}-d_{x^{2}-y^{2}}$ orbitals.

Supported by the small magnetic field on the $\mathrm{Ni}$ atoms an imbalance appears between the $\uparrow \uparrow$ and $\downarrow \downarrow$ triplet components: the charge associated to the $\uparrow \uparrow$ triplet component on the $\mathrm{Ni}$ atom was larger by $9 \%$ compared to the $\downarrow \downarrow$ component, which means that the above states break the time-reversal symmetry and give a non-unitary pairing state as found in reference [26].

By calculating the DOS for finite temperatures the specific heat can be obtained as $C=$ $\left.\left.-T \frac{\partial}{\partial T} \int d \varepsilon D_{T}(\varepsilon)\left[f_{T}(\varepsilon) \log f_{T}(\varepsilon)+f_{T}(-\varepsilon)\right) \log f_{T}(-\varepsilon)\right)\right]$, where $f_{T}(\varepsilon)$ is the Fermi-Dirac distribution and $D_{T}(\varepsilon)$ is the temperature dependent DOS. The results are shown in Figure 7 where we compare our results with experimental measurements [21]. The pairing model $\left(\mathrm{d}_{z x}-\mathrm{d}_{z y}\right.$ orbital) with nodal gap structure clearly does not fit to the experimental one and it has a power law $\left(C \sim T^{2}\right)$ behavior at low temperatures (which is the consequence of the line nodes). However, the fully gapped scenarios are in good agreement with the experimental one and both of them have exponential behavior at low temperatures. Since the equal-spin pairing model on the $d_{z^{2}}-d_{x^{2}-y^{2}}$ orbitals are closer to the experimental specific heat and it needs a lower interaction strength, we may conclude that this pairing model is the most likely to happen. The results are robust against reasonably small perturbations $(<5 \%)$ coming from other pairing channels. The local attraction between equal spins could be a consequence of Hund's rule coupling [57], or, alternatively, it can also be mediated by phonons. However, since the Fermi surface has a strong orbital degeneracy, we can not exclude the possibility of a more complicated pairing interaction where more orbitals are involved.

In summary, we have presented a first-principles based semiphenomenological approach to study the superconductivity in the non-centrosymmetric superconductor $\mathrm{LaNiC}_{2}$ within a fully relativistic description. We have found that the singlet-triplet mixing effect due to spinorbit coupling is only modest, which confirms the results of earlier group theoretical findings [26]. We have assumed interorbital equal-spin pairing on the $\mathrm{Ni}$ atoms, and it was proven that it breaks time-reversal symmetry and most of these scenarios yield line nodes in the quasiparticle particle spectrum, however two of them were corresponding to fully gapped spectrum (this behavior was linked to the noncentrosymmetric crystal structure). The equalspin pairing model on the $d_{z^{2}}-d_{x^{2}-y^{2}}$ orbitals could fit most accurately the experimental specific heat. While the above theory clearly contains a phenomenologically fitted parameter, it provides a new ground for speculations about the pairing mechanism. However, it should be mentioned that in the last decade enormous progress has been made in the fully ab-initio theory of superconductivity including both conventional $[31,32,58,59]$ and unconventional $[60,61]$ superconductors, which makes possible to get of rid of every phenomenological parameters.

This work was supported by EPSRC through the project "Unconventional Superconductors: New paradigms for new materials" under EP/P007392/1, and the Hungarian National Research, Development and Innovation Office under the contracts No. K115632. The authors would like to thank the fruitful discussions to Jorge Quintanilla, Hubert Ebert, and Martin Gradhand.

\section{Author contribution statement}

All authors contributed significantly to this work. All authors were involved in the preparation of this manuscript, and have read and approved its final version.

Open Access This is an open access article distributed under the terms of the Creative Commons Attribution License (http://creativecommons.org/licenses/by/4.0), which permits unrestricted use, distribution, and reproduction in any medium, provided the original work is properly cited.

\section{References}

1. M. Smidman, M.B. Salamon, H.Q. Yuan, D.F. Agterberg, Rep. Prog. Phys. 80, 036501 (2017)

2. A.D. Hillier, J. Quintanilla, R. Cywinski, Phys. Rev. Lett. 102, 117007 (2009)

3. R.H. Heffner, J.L. Smith, J.O. Willis, P. Birrer, C. Baines, F.N. Gygax, B. Hitti, E. Lippelt, H.R. Ott, A. Schenck, E.A. Knetsch, J.A. Mydosh, D.E. MacLaughlin, Phys. Rev. Lett. 65, 2816 (1990)

4. G.M. Luke, Y. Fudamoto, K.M. Kojima, M.I. Larkin, J. Merrin, B. Nachumi, Y.J. Uemura, Y. Maeno, Z.Q. Mao, Y. Mori, H. Nakamura, M. Sigrist, Nature 394, 558 (1998)

5. G.M. Luke, A. Keren, L.P. Le, W.D. Wu, Y.J. Uemura, D.A. Bonn, L. Taillefer, J.D. Garrett, Phys. Rev. Lett. 71, 1466 (1993)

6. Y. Aoki, A. Tsuchiya, T. Kanayama, S.R. Saha, H. Sugawara, H. Sato, W. Higemoto, A. Koda, K. Ohishi, K. Nishiyama, R. Kadono, Phys. Rev. Lett. 91, 067003 (2003) 
7. L. Shu, W. Higemoto, Y. Aoki, A.D. Hillier, K. Ohishi, K. Ishida, R. Kadono, A. Koda, O.O. Bernal, D.E. MacLaughlin, Y. Tunashima, Y. Yonezawa, S. Sanada, D. Kikuchi, H. Sato, H. Sugawara, T.U. Ito, M.B. Maple, Phys. Rev. B 83, 100504 (2011)

8. A. Maisuradze, W. Schnelle, R. Khasanov, R. Gumeniuk, M. Nicklas, H. Rosner, A. Leithe-Jasper, Y. Grin, A. Amato, P. Thalmeier, Phys. Rev. B 82, 024524 (2010)

9. A.D. Hillier, J. Quintanilla, B. Mazidian, J.F. Annett, R. Cywinski, Phys. Rev. Lett. 109, 097001 (2012)

10. P.K. Biswas, H. Luetkens, T. Neupert, T. Stürzer, C. Baines, G. Pascua, A.P. Schnyder, M.H. Fischer, J. Goryo, M.R. Lees, H. Maeter, F. Brückner, H.-H. Klauss, M. Nicklas, P.J. Baker, A.D. Hillier, M. Sigrist, A. Amato, D. Johrendt, Phys. Rev. B 87, 180503 (2013)

11. R.P. Singh, A.D. Hillier, B. Mazidian, J. Quintanilla, J.F. Annett, D.M. Paul, G. Balakrishnan, M.R. Lees, Phys. Rev. Lett. 112, 107002 (2014)

12. D.A. Mayoh, J.A.T. Barker, R.P. Singh, G. Balakrishnan, D.M. Paul, M.R. Lees, Phys. Rev. B 96, 064521 (2017)

13. T. Shang, G.M. Pang, C. Baines, W.B. Jiang, W. Xie, A. Wang, M. Medarde, E. Pomjakushina, M. Shi, J. Mesot, H.Q. Yuan, T. Shiroka, Phys. Rev. B 97, 020502 (2018)

14. A. Bhattacharyya, D.T. Adroja, J. Quintanilla, A.D. Hillier, N. Kase, A.M. Strydom, J. Akimitsu, Phys. Rev. B 91, 060503 (2015)

15. J.A.T. Barker, D. Singh, A. Thamizhavel, A.D. Hillier, M.R. Lees, G. Balakrishnan, D.M. Paul, R.P. Singh, Phys. Rev. Lett. 115, 267001 (2015)

16. T. Watashige, Y. Tsutsumi, T. Hanaguri, Y. Kohsaka, S. Kasahara, A. Furusaki, M. Sigrist, C. Meingast, T. Wolf, H. Löhneysen, T. Shibauchi, Y. Matsuda, Phys. Rev. X 5, 031022 (2015)

17. W. Lee, H. Zeng, Y. Yao, Y. Chen, Physica C 266, 138 (1996)

18. I. Bonalde, R.L. Ribeiro, K.J. Syu, H.H. Sung, W.H. Lee, New J. Phys. 13, 123022 (2011)

19. J.F. Landaeta, D. Subero, P. Machado, F. Honda, I. Bonalde, Phys. Rev. B 96, 174515 (2017)

20. Y. Hirose, T. Kishino, J. Sakaguchi, Y. Miura, F. Honda, T. Takeuchi, E. Yamamoto, Y. Haga, H. Harima, R. Settai, Y. Ōnuki, J. Phys. Soc. Jpn. 81, 113703 (2012)

21. J. Chen, L. Jiao, J.L. Zhang, Y. Chen, L. Yang, M. Nicklas, F. Steglich, H.Q. Yuan, New J. Phys. 15, 053005 (2013)

22. Y. Iwamoto, Y. Iwasaki, K. Ueda, T. Kohara, Phys. Lett. A 250, 439 (1998)

23. S. Katano, K. Shibata, K. Nakashima, Y. Matsubara, Phys. Rev. B 95, 144502 (2017)

24. J. Chen, L. Jiao, J.L. Zhang, Y. Chen, L. Yang, H.Q. Yuan, J. Korean Phys. Soc. 63, 463 (2013)

25. A. Sumiyama, D. Kawakatsu, J. Gouchi, A. Yamaguchi, G. Motoyama, Y. Hirose, R. Settai, Y. Ōnuki, J. Phys. Soc. Jpn. 84, 013702 (2015)

26. J. Quintanilla, A.D. Hillier, J.F. Annett, R. Cywinski, Phys. Rev. B 82, 174511 (2010)

27. B.L. Gyorffy, Z. Szotek, W.M. Temmerman, O.K. Andersen, O. Jepsen, Phys. Rev. B 58, 1025 (1998)

28. K. Capelle, E.K.U. Gross, Phys. Rev. B 59, 7140 (1999)

29. K. Capelle, E.K.U. Gross, Phys. Rev. B 59, 7155 (1999)

30. G. Csire, A. Deák, B. Nyári, H. Ebert, J.F. Annett, B. Újfalussy, Phys. Rev. B 97, 024514 (2018)

31. A. Linscheid, A. Sanna, F. Essenberger, E.K.U. Gross, Phys. Rev. B 92, 024505 (2015)
32. A. Linscheid, A. Sanna, E.K.U. Gross, Phys. Rev. B 92, 024506 (2015)

33. M.A.L. Marques, M. Lüders, N.N. Lathiotakis, G. Profeta, A. Floris, L. Fast, A. Continenza, E.K.U. Gross, S. Massidda, Phys. Rev. B 72, 024546 (2005)

34. A. Floris, G. Profeta, N.N. Lathiotakis, M. Lüders, M.A.L. Marques, C. Franchini, E.K.U. Gross, A. Continenza, S. Massidda, Phys. Rev. Lett. 94, 037004 (2005)

35. A. Floris, A. Sanna, S. Massidda, E.K.U. Gross, Phys. Rev. B 75, 054508 (2007)

36. C. Bersier, A. Floris, P. Cudazzo, G. Profeta, A. Sanna, F. Bernardini, M. Monni, S. Pittalis, S. Sharma, H. Glawe, A. Continenza, S. Massidda, E.K.U. Gross, J. Phys.: Condens. Matter 21, 164209 (2009)

37. S. Massidda, F. Bernardini, C. Bersier, A. Continenza, P. Cudazzo, A. Floris, H. Glawe, M. Monni, S. Pittalis, G. Profeta, A. Sanna, S. Sharma, E.K.U. Gross, Supercond. Sci. Technol. 22, 034006 (2009)

38. A. Sanna, G. Profeta, S. Massidda, E.K.U. Gross, Phys. Rev. B 86, 014507 (2012)

39. J.A. Flores-Livas, M. Amsler, C. Heil, A. Sanna, L. Boeri, G. Profeta, C. Wolverton, S. Goedecker, E.K.U. Gross, Phys. Rev. B 93, 020508 (2016)

40. A.J. Flores-Livas, A. Sanna, K.E. Gross, Eur. Phys. J. B 89, 63 (2016)

41. W.M. Temmerman, Z. Szotek, B.L. Gyorffy, O.K. Andersen, O. Jepsen, Phys. Rev. Lett. 76, 307 (1996)

42. Z. Szotek, B.L. Gyorffy, W.M. Temmerman, O.K. Andersen, O. Jepsen, J. Phys.: Condens. Matter 13, 8625 (2001)

43. G. Csire, B. Újfalussy, J. Cserti, B. Győrffy, Phys. Rev. B 91, $165142(2015)$

44. G. Csire, J. Cserti, B. Újfalussy, J. Phys.: Condens. Matter 28, 495701 (2016)

45. G. Csire, S. Schönecker, B. Újfalussy, Phys. Rev. B 94, $140502(2016)$

46. A. Subedi, D.J. Singh, Phys. Rev. B 80, 094413 (2009)

47. J. Laverock, T.D. Haynes, C. Utfeld, S.B. Dugdale, Phys. Rev. B 80, 125111 (2009)

48. I. Hase, T. Yanagisawa, J. Phys. Soc. Jpn. 78, 084724 (2009)

49. T. Yanagisawa, I. Hase, J. Phys. Soc. Jpn. 81, SB039 (2012)

50. B. Wiendlocha, R. Szczśniak, A.P. Durajski, M. Muras, Phys. Rev. B 94, 134517 (2016)

51. P. Giannozzi, S. Baroni, N. Bonini, M. Calandra, R. Car, C. Cavazzoni, D. Ceresoli, G.L. Chiarotti, M. Cococcioni, I. Dabo, A.D. Corso, S. de Gironcoli, S. Fabris, G. Fratesi, R. Gebauer, U. Gerstmann, C. Gougoussis, A. Kokalj, M. Lazzeri, L. Martin-Samos, N. Marzari, F. Mauri, R. Mazzarello, S. Paolini, A. Pasquarello, L. Paulatto, C. Sbraccia, S. Scandolo, G. Sclauzero, A.P. Seitsonen, A. Smogunov, P. Umari, R.M. Wentzcovitch, J. Phys.: Condens. Matter 21, 395502 (2009)

52. S. Katano, H. Nakagawa, K. Matsubayashi, Y. Uwatoko, H. Soeda, T. Tomita, H. Takahashi, Phys. Rev. B 90, 220508 (2014)

53. W. Schäfer, G. Will, J. Yakinthos, P. Kotsanidis, J. Alloys Compd. 180, 251 (1992)

54. V.P. Antropov, M.I. Katsnelson, M. van Schilfgaarde, B.N. Harmon, Phys. Rev. Lett. 75, 729 (1995)

55. H. Ebert, H. Freyer, A. Vernes, G.-Y. Guo, Phys. Rev. B 53, 7721 (1996) 
56. Z.F. Weng, J.L. Zhang, M. Smidman, T. Shang, J. Quintanilla, J.F. Annett, M. Nicklas, G.M. Pang, L. Jiao, W.B. Jiang, Y. Chen, F. Steglich, H.Q. Yuan, Phys. Rev. Lett. 117, 027001 (2016)

57. A. Georges, L. de Medici, J. Mravlje, Annu. Rev. Condens. Matter Phys. 4, 137 (2013)

58. M. Lüders, M.A.L. Marques, N.N. Lathiotakis, A. Floris, G. Profeta, L. Fast, A. Continenza, S. Massidda, E.K.U. Gross, Phys. Rev. B 72, 024545 (2005)
59. M.A.L. Marques, M. Lüders, N.N. Lathiotakis, G. Profeta, A. Floris, L. Fast, A. Continenza, E.K.U. Gross, S. Massidda, Phys. Rev. B 72, 024546 (2005)

60. F. Essenberger, A. Sanna, A. Linscheid, F. Tandetzky, G. Profeta, P. Cudazzo, E.K.U. Gross, Phys. Rev. B 90, $214504(2014)$

61. F. Essenberger, A. Sanna, P. Buczek, A. Ernst, L. Sandratskii, E.K.U. Gross, Phys. Rev. B 94, 014503 (2016) 